\title{
Precision measurements of the top quark couplings at the FCC
}

Patrick Janot ${ }^{* \dagger}$

CERN, Geneva, Switzerland

E-mail: patrick.janotecern.ch

The design study of the Future Circular Colliders (FCC) in a 100-km ring in the Geneva area has started at CERN at the beginning of 2014, as an option for post-LHC particle accelerators. The study has an emphasis on proton-proton and electron-positron high-energy frontier machines. In the current plans, the first step of the FCC physics programme would exploit a high-luminosity $\mathrm{e}^{+} \mathrm{e}^{-}$collider called FCC-ee, with centre-of-mass energies ranging from below the $Z$ pole to the $\mathrm{t} \overline{\mathrm{t}}$ threshold and beyond, followed by $100 \mathrm{TeV}$ proton-proton collisions as ultimate goal. When combined, these two steps offer a large palette of complementary measurements and sensitivity for new physics. In particular, the association of the FCC-ee and the FCC-hh allows measurements of the top-quark electroweak and Yukawa couplings to be performed with unrivaled precision.

The European Physical Society Conference on High Energy Physics

22-29 July 2015

Vienna, Austria

*Speaker.

${ }^{\dagger}$ On behalf of the FCC Design Study Group. 


\section{The physics programme of the FCC-ee}

The core physics programme $[1,2]$ of the future high-luminosity $\mathrm{e}^{+} \mathrm{e}^{-}$circular collider $($FCCee) includes precision measurements at centre-of-mass energies from 90 to $350 \mathrm{GeV}$ :

(i) a scan of the $\mathrm{Z}$ pole [3], enabling the measurements of, e.g., $m_{\mathrm{Z}}$ and $\Gamma_{\mathrm{Z}}$ to better than $100 \mathrm{keV}$, $\sin ^{2} \theta_{W}$ to $5 \times 10^{-6}, \alpha_{S}\left(m_{\mathrm{Z}}\right)$ to $2 \times 10^{-4}, \alpha_{\mathrm{QED}}\left(m_{\mathrm{Z}}\right)$ to $2 \times 10^{-5} \ldots$, as well as a unique programme of rare process searches and flavour physics with up to $10^{13} \mathrm{Z}$ decays [4];

(ii) a scan of the WW threshold [3] towards the measurement of the W mass to $300 \mathrm{keV}$;

(iii) a Higgs factory [5], for a tenfold improvement of the HL-LHC precision on the Higgs couplings, a measurement of the total width to $1 \%$, and a constraint on the invisible branching ratio at the per-mil level;

(iv) a scan of the top threshold, for a measurement of the top-quark mass [3] with a statistical precision of the order of $10 \mathrm{MeV}$.

If these target precisions are reached and no deviations with respect to the standard model predictions are observed, a global fit to all these masurements [6] will set constraints on weaklycoupled new physics up to a scale of $\sim 100 \mathrm{TeV}$, and on news physics coupled to the Higgs sector up to $\sim 10 \mathrm{TeV}$. This sensitivity to new physics matches and even extends the direct new physics discovery potential of the FCC-hh.

This fascinating perspective is made possible by the unique levels of luminosity promised by circular colliders [7] collected by up to four experiments. The luminosities and the numbers of events of a given type expected at each centre-of-mass energies for a FCC-ee year of running with four interaction points are summarized in Table 1. In this configuration, the core physics targets of the FCC-ee programme would be met in 8 to 10 years at full luminosity, after the commissioning period.

\begin{tabular}{|l|c|c|c|c|c|}
\hline$\sqrt{s}(\mathrm{GeV})$ & 90 & 160 & 240 & 350 & $350+$ \\
\hline $\mathscr{L}\left(\mathrm{ab}^{-1} /\right.$ year $)$ & 86.0 & 15.2 & 3.5 & 1.0 & 1.0 \\
\hline Events/year & $3.6 \times 10^{12}$ & $6.1 \times 10^{7}$ & $7.0 \times 10^{5}$ & $4.2 \times 10^{5}$ & $2.5 \times 10^{4}$ \\
\hline Event type & $\mathrm{Z}$ & $\mathrm{WW}$ & $\mathrm{HZ}$ & $\mathrm{t}$ & $\mathrm{WW} \rightarrow \mathrm{H}$ \\
\hline \hline Years & $0.3(2.5)$ & 1 & 3 & 0.5 & 3 \\
\hline
\end{tabular}

Table 1: Integrated luminosities in $\mathrm{ab}^{-1}$ and numbers of events expected for each year of running at the FCC-ee. The last row indicates the time needed to complete the core FCC-ee physics programme with $10^{12}$ $\left(10^{13}\right)$ events at the $\mathrm{Z}$ pole. In this table, each year amounts to $10^{7}$ seconds of data taking.

In this report, the FCC capability to measure the top-quark couplings is investigated. In particular, the sensitivity of the FCC-ee to the top-quark electroweak couplings is shown to be excellent for a centre-of-mass energy just above the $t \bar{t}$ threshold and without the benefit of longitudinally polarized beams. The expected precision on these couplings can be then used to extract the ttH coupling at the FCC-hh with a per-cent-level precision. The FCC-ee targets are compared with projections from the ILC in the "H20" design running scenario [8], as is done in Ref. [9]. This running scenario invokes a similar running time (about 8 to 10 years), albeit with a slightly more optimistic definition of a "year" $\left(1.6 \times 10^{7}\right.$ seconds of data taking $)$ and a preliminary modelling of the commissioning time. 


\section{The top-quark electroweak couplings at the FCC-ee}

The measurement of the top-quark electroweak couplings was, originally, not part of the FCCee core physics programme. Indeed, this measurement was claimed [10] to require both a centreof-mass energy well beyond the top-pair production threshold and a large longitudinal polarization of the incoming $\mathrm{e}^{ \pm}$beams. These claims were recently revisited [11] in the context of the FCC-ee.

In $\mathrm{e}^{+} \mathrm{e}^{-}$collisions, the availability of initial state longitudinal polarization provides enhanced sensitivity to the initial-state and, sometimes, final-state couplings to the photon and the $\mathrm{Z}$ boson. Similar information can often be obtained, however, from the final-state polarization, as is the case in the $\mathrm{e}^{+} \mathrm{e}^{-} \rightarrow \gamma^{*} / \mathrm{Z} \rightarrow \mathrm{tt}$ production at the FCC-ee: in this process, anomalous couplings of the top quark to the $\mathrm{Z}$ and to the photon would alter the top-quark polarization. This anomalous polarization is maximally transferred to the top-quark decay products via the weak decay $\mathrm{t} \rightarrow \mathrm{Wb}$, leading to a modification of the final kinematics, and in particular of the angular and energy distributions of the leptons from the $\mathrm{W}$ decays. The large luminosity of the FCC-ee allows in turn to extract precise measurements of the anomalous couplings from these distributions. A similar situation was encountered at LEP, where the measurement of the $\mathrm{Z} \rightarrow \tau^{+} \tau^{-}$event rate and of the $\tau$ polarization sufficed to determine the couplings of the $\tau$ to the $\mathrm{Z}$, regardless of the initial state polarization.

The angular and energy distributions of the leptons from $\mathrm{e}^{+} \mathrm{e}^{-} \rightarrow \mathrm{t} \overline{\mathrm{t}} \rightarrow \mathrm{b} \overline{\mathrm{b}} \mathrm{q} \overline{\mathrm{q}}^{\prime} \ell v_{\ell}(\ell=\mathrm{e}, \mu)$ have been determined analytically in Ref. [12] as a function of the centre-of-mass energy and of the incoming beam polarization, in presence of anomalous $\mathrm{tt} \gamma$ and $\mathrm{ttZ}$ couplings, denoted $\delta A_{\gamma, \mathrm{Z}}$, $\delta B_{\gamma, \mathrm{Z}}, \delta C_{\gamma, \mathrm{Z}}$, and $\delta D_{\gamma, \mathrm{Z}}$. These anomalous couplings modify the ttV vertex $(V=\gamma, \mathrm{Z})$ as follows:

$$
\Gamma_{t t V}^{\mu}=\frac{g}{2}\left[\gamma^{\mu}\left\{\left(A_{V}+\delta A_{V}\right)-\gamma_{5}\left(B_{V}+\delta B_{V}\right)\right\}+\frac{\left(p_{t}-p_{\bar{t}}\right)^{\mu}}{2 m_{\mathrm{t}}}\left(\delta C_{V}-\delta D_{V} \gamma_{5}\right)\right]
$$

where $A_{V}$ and $B_{V}$ are the vector and axial standard model top couplings, and modify the lepton angular and energy distribtution as sketched in Fig. 1.
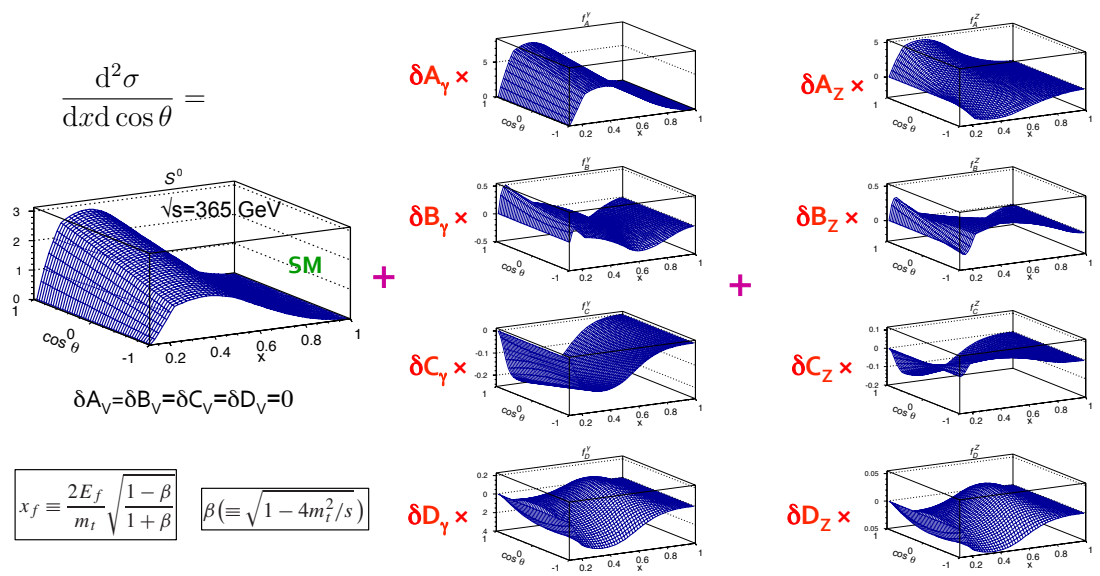

Figure 1: The differential production cross section $\mathrm{d}^{2} \sigma / \mathrm{d} \cos \theta \mathrm{d} x_{f}$ for $\mathrm{e}^{+} \mathrm{e}^{-} \rightarrow \mathrm{t} \overline{\mathrm{t}} \rightarrow \mathrm{b} \overline{\mathrm{b}} \mathrm{q} \bar{q}^{\prime} \ell v_{\ell}(\ell=\mathrm{e}, \mu)$ at $\sqrt{s}=365 \mathrm{GeV}$, as a function of the cosine of the lepton polar angle $\cos \theta$ and the reduced lepton energy $x_{f}$ in the standard model (left). The additive modifications arising from anomalous $\mathrm{tt} \gamma$ and $\mathrm{ttZ}$ couplings are displayed on the right. 
It is shown in Ref. [11] that a likelihood fit of the anomalous couplings to the double differential cross section is statistically optimal at the FCC-ee for $\sqrt{s}=365 \mathrm{GeV}$, without beam polarization. With conservative assumptions on lepton identification, b-tagging efficiencies, and lepton angular and momentum resolutions, it is estimated therein that absolute precisions of the order of $1[3] \times 10^{-3}\left(1[2] \times 10^{-2}\right)$ can be expected after three FCC-ee years at $\sqrt{s}=365 \mathrm{GeV}$ on $\delta A_{\gamma[\mathrm{Z}]}$ $\left(\delta B_{\gamma[Z]}\right)$, if $\delta C_{\gamma[Z]}=0$. A full simulation of the CLIC/ILD detector operated in the FCC-ee environment [13] has recently confirmed these analytical preliminary estimates.

These target precisions will be met only if the theoretical prediction for the top-pair cross section can be kept under control to better than $\pm 2 \%$, perhaps a serious challenge only $20 \mathrm{GeV}$ above the production threshold. It is inferred in Ref. [14], however, that the total theoretical uncertainty is, already today, at the level of $\pm 4 \%$ at $\sqrt{s}=365 \mathrm{GeV}$. A factor 2 improvement might be beyond the current state of the art, but is probably within reach on the time scale required by the FCC-ee.

The sensitivity of these projections to new physics is evaluated under the mild additional assumption that the electric charge of the top quark is $+2 / 3$. New physics information is then entirely contained in the ttZ axial and vector couplings $A_{\mathrm{Z}}$ and $B_{\mathrm{Z}}$ or, equivalently ( as is done for example in Ref. [15]), in the couplings to the left-handed and right-handed top quark, $g_{L}$ and $g_{R}$, trivially related to $A_{\mathrm{Z}}$ and $B_{\mathrm{Z}}$ :

$$
g_{R}+g_{L}=g A_{\mathrm{Z}} \text { and } g_{R}-g_{L}=g B_{\mathrm{Z}},
$$

where $g$ is the weak coupling constant. The relative precision expected at FCC-ee for $g_{L}$ and $g_{R}$ is displayed in Fig. 2, and is compared to the projections made for the LHC, the HL-LHC [16] and the ILC $[9,17]$. The most exotic new physics models - represented by purple dots in Fig. 2 - will
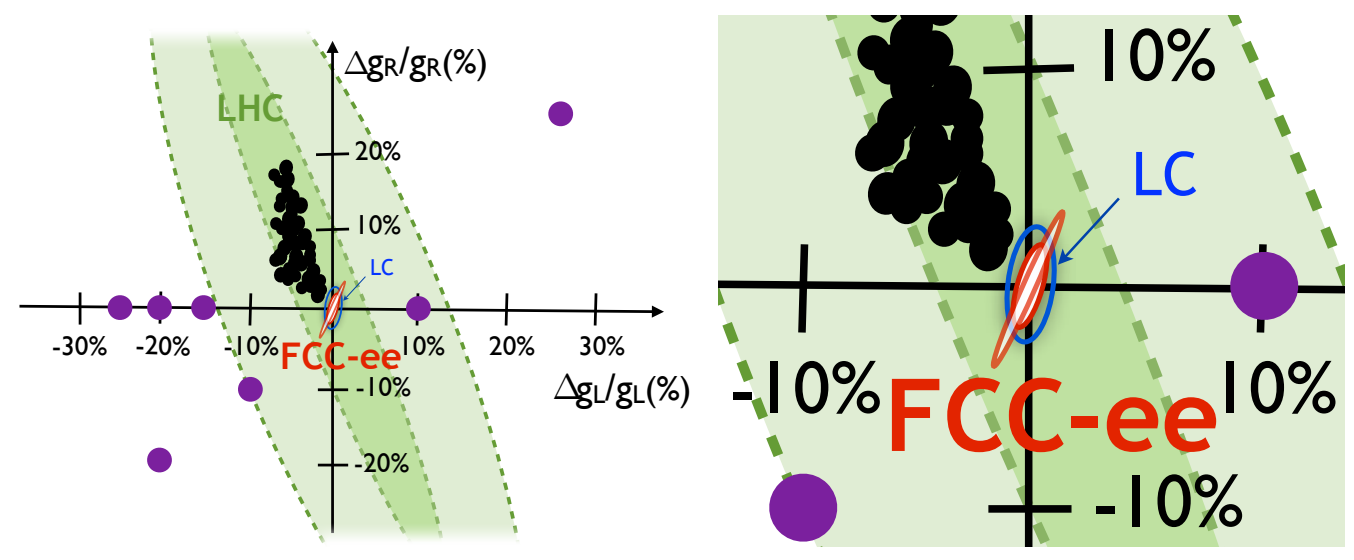

Figure 2: (Adapted from Ref. [15]) Expected relative precision on the $\mathrm{Zt}_{\mathrm{L}} \mathrm{t}_{\mathrm{L}}$ and $\mathrm{Z} \mathrm{t}_{\mathrm{R}} \mathrm{t}_{\mathrm{R}}$ couplings at the LHC (lighter green), the HL-LHC (darker green), the ILC (blue) and the FCC-ee (orange, red). The left plot is a zoom of the right plot in the $\pm 10 \%$ window. Typical deviations from the standard model in various new physics models are represented by the purple dots. The black dots indicate the deviations expected for different parameter choices of 4D Composite Higgs Models, with $f<2 \mathrm{TeV}$. For the FCC-ee, the orange ellipse is obtained from an analysis of the lepton angular and energy distributions, while the smaller red ellipse is obtained if the angular and energy distributions of the $b$ jets can be exploited.

already be explored by the modest precision expected at the HL-LHC, but an $\mathrm{e}^{+} \mathrm{e}^{-}$collider will be needed to disentangle the standard model from, e.g., 4D composite Higgs models (4DCHM, black 
dots). If the characteristic scale of these models, $f$, is constrained to be smaller than $2 \mathrm{TeV}$, as is the case in Fig. 2, the FCC-ee will be able to discover them from the sole analysis of the lepton angular and energy distributions in top-quark decays with a $5 \sigma$ significance. The significance expected at the ILC, from the analysis of the top forward-backward asymmetry and the total rate, with two different beam polarization configurations $\left(\mathscr{P}_{-}= \pm 0.8\right.$ and $\left.\mathscr{P}_{+}=\mp 0.3\right)$, would be limited to $1.5 \sigma$.

It is important to realize, however, that top-quark couplings might not be the only quantities affected by new physics. Composite Higgs models, in particular, affect Higgs couplings as well. The deviations expected on the Higgs couplings to the $\mathrm{Z}$ boson and to the $\mathrm{b}$ quark for the same set of 4DHCM as in Fig. 2 are displayed in Fig. 3, and compared to the corresponding relative precisions expected at the HL-LHC [5], the ILC [18] and the FCC-ee [1]. From these Higgs coupling measurements, the FCC-ee will be able to discover these models with a $10 \sigma$ significance (to be compared to about $2 \sigma$ for the ILC), complementary to, and possibly earlier than, the top electroweak coupling measurements.

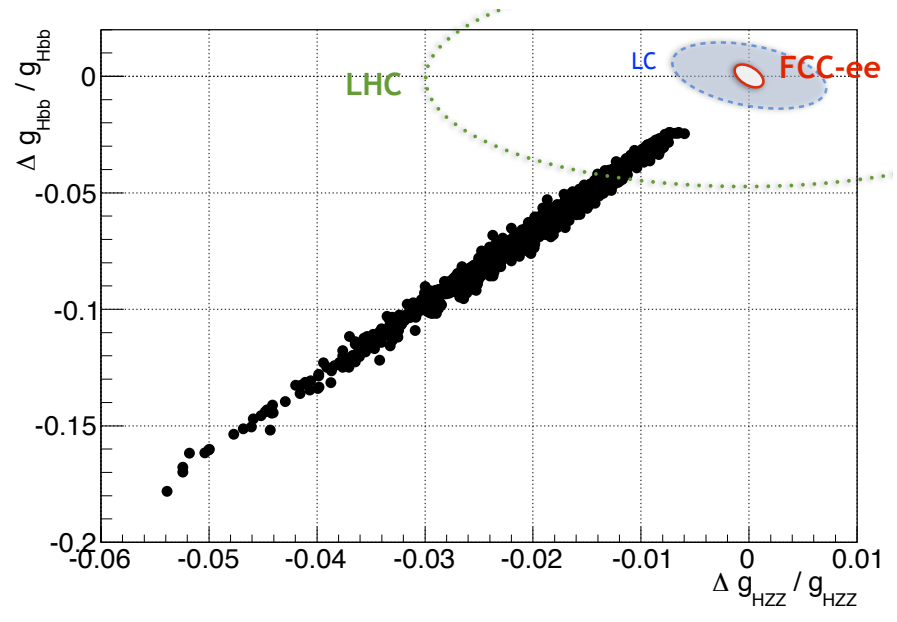

Figure 3: Expected relative precision on the HZZ and Hbb couplings at the HL-LHC (dotted green), the ILC (dashed blue) and the FCC-ee (full red). The black dots indicate the deviations expected for the same parameter choices of 4D Composite Higgs Models $(f<2 \mathrm{TeV})$ as in Fig. 2.

\section{The top-quark Yukawa couping at the FCC-hh}

New physics is also expected to show up in top-quark Yukawa coupling to the Higgs boson. For the previous set of composite Higgs models, deviations similar to or slightly smaller than those of the b-quark Yukawa couplings are expected, typically of the order of 1 to $2 \%$ for $f=2 \mathrm{TeV}$. It has been shown [19] that $\mathrm{e}^{+} \mathrm{e}^{-}$colliders with centre-of-mass energy significantly larger than the FCC-ee or the ILC are needed to measure the top-quark Yukawa couplings with a precision significantly smaller than $10 \%$ : for $\sqrt{s}=1$ to $3 \mathrm{TeV}$, a precision of 2 to $4 \%$ can be contemplated, which still does not suffice to challenge these models. As shown in Table 2, a similar performance is expected from the HL-LHC, with $3 \mathrm{ab}^{-1}$ at $\sqrt{s}=13 \mathrm{TeV}$. 
The FCC opens a unique window for this measurement, with a combination of the FCCee and the FCC-hh data [20]. In pp collisions, the $\mathrm{pp} \rightarrow \mathrm{t} \overline{\mathrm{t}} \mathrm{H}$ cross section increases by a factor 70 - from 0.47 to $33.2 \mathrm{pb}$ - when the centre-of-mass energy goes from 13 to $100 \mathrm{TeV}$. With a target integrated luminosity of $20 \mathrm{ab}^{-1}$, almost a billion t $\overline{\mathrm{t}} \mathrm{H}$ events are expected to be produced at the FCC-hh, leading to a negligible statistical uncertainty on the top Yukawa coupling. The measurement of the titH cross section would therefore be only limited by systematic uncertainties, dominated by the renormalization/factorization scale and PDF uncertainties at the level of $\pm 10 \%$. These uncertainties almost exactly cancel in the ratio $\sigma(\mathrm{tt} H) / \sigma(\mathrm{tt} Z) \simeq 0.6$ to better than $\pm 2 \%$ at $\sqrt{s}=100 \mathrm{TeV}$. A measurement of this ratio amounts to measuring the quantity $R$ defined by

$$
R=\frac{\lambda_{\mathrm{t}}^{2}}{g_{R}^{2}+g_{L}^{2}} \times \frac{\mathrm{BR}\left(\mathrm{H} \rightarrow \mathrm{b} \overline{\mathrm{b}}, \mathrm{ZZ}, \tau^{+} \tau^{-}, \ldots\right)}{\mathrm{BR}\left(\mathrm{Z} \rightarrow \ell^{+} \ell^{-}\right)},
$$

where $\lambda_{\mathrm{t}}$ is the top-quark Yukawa coupling and $g_{L, R}$ are the top-quark weak couplings to the $\mathrm{Z}$ boson. The first ratio of Eq. 3.1 can be predicted, already today, with an accuracy better than $2 \%$, and the FCC-ee can deliver a measurement of $g_{L}^{2}+g_{R}^{2}$ with a precision of $\sim 2 \%$, as can be deduced from Fig. 2. For all practical purposes, the second ratio is perfectly known, as the Higgs branching fractions can be measured in a model-independent way at the FCC-ee with a sub-per-cent precision, and the $\mathrm{Z}$ leptonic branching ratios will be known with a precision better than $10^{-5}$. A combination of all these measurements therefore allows the value of top-quark Yukawa coupling to be inferred with an accuracy of $\sim 1.5 \%$ at the FCC.

These projections are summarized in Table 2, both for the top-quark Yukawa and for the Higgs trilinear self-coupling [5], another important parameter to be measured at future colliders.

\begin{tabular}{|c|c|c|c|c|}
\hline Collider & HL-LHC & ILC & LC 1-3 TeV & FCC-ee+hh \\
\hline$\lambda_{\mathrm{t}}$ & $4 \%$ & $14 \%$ & $2-4 \%$ & $1-2 \%$ \\
\hline$\lambda_{\mathrm{H}}$ & $50 \%$ & $83 \%$ & $10-15 \%$ & $5-10 \%$ \\
\hline
\end{tabular}

Table 2: Expected precision on the top-quark Yukawa coupling (first row) and, for completeness, on the Higgs trilinear self-coupling (second row) at the HL-LHC, the ILC, a linear $\mathrm{e}^{+} \mathrm{e}^{-}$collider with $\sqrt{s}=1$ to $3 \mathrm{TeV}$, and the FCC - with a combination of the FCC-ee and the FCC-hh data.

\section{Conclusion}

The measurement of the angular and energy distributions in semi-leptonic $t \bar{t}$ events at the FCC-ee has a strong potential for a precise determination of the top-quark electroweak couplings. The optimal centre-of-mass energy for this measurement is just above the tit threshold, typically $\sqrt{s}=365 \mathrm{GeV}$. The lack of beam polarization is compensated by the polarization of the top quarks, and by a large integrated luminosity. In combination with this measurement and those of the Higgs boson branching fractions at the FCC-ee, the large $\bar{t} \mathrm{t} H$ and $\mathrm{t} \bar{Z}$ production cross sections at the FCC-hh, as well as the cancellation of the dominant theory uncertainties in their ratio, allow the top-quark Yukawa coupling to be measured with a per-cent level precision.

Taken in isolation, these measurements at the FCC are already more sensitive to the presence of new physics than those at other collider options considered so far. New physics responsible 
for anomalous top couplings is also likely to affect the properties of the $\mathrm{Z}, \mathrm{W}$, and Higgs bosons. The FCC will therefore also have many opportunities for new physics discoveries, as well as a palette of precision measurements to help identify the underlying theory, through a global fit to these properties and their correlations.

The combination of the FCC-ee and the FCC-hh offers, for a great cost effectiveness, the best precision and the best search reach of all options presently on the market.

\section{References}

[1] M. Bicer et. al., "First look at the physics case of TLEP", Journal of High Energy Physics 01 (2014) 164

[2] A. Blondel, "Physics at the FCC-ee", poster presented at EPS-HEP2015, Vienna, 22-29 July 2015, in these proceedings

[3] M. Dam, "Precision electroweak physics at the FCC", talk presented at EPS-HEP2015, Vienna, 22-29 July 2015 , in these proceedings

[4] S. Monteil, "Flavours at the FCC-ee", talk presented at EPS-HEP2015, Vienna, 22-29 July 2015, in these proceedings

[5] M. Klute, "Higgs physics at the FCC", talk presented at EPS-HEP2015, Vienna, 22-29 July 2015, in these proceedings

[6] J. Ellis and T. You, "Sensitivities of prospective future $\mathrm{e}^{+} \mathrm{e}^{-}$colliders to decoupled new physics", arXiv:1510.04561 (2015).

[7] M. Koratzinos, "FCC-ee energy and luminosity", poster presented at EPS-HEP2015, Vienna, 22-29 July 2015 , in these proceedings

[8] T. Barklow et. al., "ILC operating scenarios", arXiv:1506.07830 (2015)

[9] M.S. Amjad et. al., "A precise characterization of the top-quark electroweak vertices at the ILC", arXiv:1505:06020 (2015)

[10] H. Baer et. al., "ILC Technical Design Report, Volume 2: Physics”, arXiv:1306:6352 (2013)

[11] P. Janot, "Top-quark electroweak couplings at the FCC-ee", Journal of High Energy Physics 04 (2015) 182

[12] B. Grazdkowski and Z. Hioki, "Optimal-observable analysis of the angular and energy distributions for top-quark decay products at polarizaed linear colliders", Nuclear Physics B585 (2000), 3; ibid, Corrigendum, Nuclear Physics B894 (2015) 585

[13] N. Foppiani and T. Pajero, "Top-quark electroweak couplings at the FCC-ee with the CLIC/ILD detector", Summer student project and laurea thesis, write-up in preparation (July-September 2015)

[14] J.R. Reuters, "WHIZARD for LC top physics", talk given at "Top physics at lepton colliders", Valencia, 30 June to 2 July 2015

[15] D. Barducci et. al., "Top pair production at a future $\mathrm{e}^{+} \mathrm{e}^{-}$machine in a composite Higgs scenario", Journal of High Energy Physics08 (2015) 127

[16] R. Roentsch and M. Schulze, "Constraining couplings of the top quarks to the $\mathrm{Z}$ boson at the LHC", Journal of High Energy Physics07 (2014) 91 
[17] R. Poeschl, “Top physics at linear colliders”, talk presented at EPS-HEP2015, Vienna, 22-29 July 2015 , in these proceedings.

[18] K. Fujii et. al., "Physics case for the international linear collider", arXiv:1506.05992 (2015)

[19] S. Dawson et. al., "Higgs Working Group report of the Snowmass 2013 community planning study", arXiv:1310.8361 (2013)

[20] M. L. Mangano et. al., "Measuring the top Yukawa coupling at 100 TeV”, arXiv:1507.08169 (2015) 